\title{
Hyperelastic behavior of modified sepiolite/SEBS thermoplastic elastomers
}

\author{
D. Perrin ${ }^{1}$, R. Léger, B. Otazaghine, P. Ienny \\ C2MA,IMT Mines Ales, 30319 Alès Cedex 9, France
}

This article has been published in Journal of Materials Science in March 17, 2017.

https://doi.org/10.1007/s10853-017-0991-z

\begin{abstract}
Thin elastomer films of styrene-ethylene-butylene-styrene block copolymer (SEBS) filled with sepiolite nanofibers nanocomposites were prepared by a dip-coating process. To increase the SEBS/Sepiolite elastomer performances, a new strategy of surface modification of sepiolite by SEBS polymer chains has been developed. In a first part the surface modification of sepiolite was characterized by FTIR and TGA. In a second part the mechanical properties of the filled SEBS films were assessed. Measurements of tensile properties and tear strength were carried to evaluate the impact of the sepiolite modification. These results are discussed in taking account the filler dispersion and the quality of the SEBS/sepiolite interface. The surface modification of the sepiolite nanofibers shows an interesting improvement of the tear strength without major modifications of SEBS matrix intrinsic hyperelastic behavior.
\end{abstract}

Keywords: thermoplastic elastomer; SEBS; hyperelastic behavior; tear strength; sepiolite; dipcoating

\section{Introduction}

Thermoplastic elastomers such as polyolefin block copolymers present a wide range of mechanical properties depending on their composition and morphology. From a microscopic point of view, they can be seen as composite materials with separated soft and rigid segments [1-3]. Hard blocks form cross-linking nodules giving the material elastomeric properties. Two cross-linking physical levels are observed with the rigid microdomains and the soft segments

\footnotetext{
${ }^{1}$ Corresponding author : didier.perrin@mines-ales.fr
} 
entanglement. Thus, by controlling the chemistry of the copolymer, nature of rigid and soft blocks and their ratio, it is possible to obtain materials with many different mechanical properties such as high elastic properties, low Young's modulus, high elongation rate [4], that can satisfy industrial demands and applications such as shoes insole, medical bags, resuscitator, etc.

Elastomers and particularly thermoplastic elastomers present a so-called Mullins effect characterized by a particular aspect of the mechanical response in filled rubbers in which the stress-strain curve depends on the maximum loading previously encountered [5-8]. Recent work [9] proposes to evaluate the theory of network alteration for the Mullins effect by reconciling both physical and phenomenological approaches using finite element applications according a modified network alteration theory of Marckmann et al. [10]. Actually, specific work input is analyzed by the integration of the stress-strain curve: the simple numerical use of the strain energy densities allows some correlations between the stiffness reduction associated to the Mullins effect, the viscoelastic response and physical considerations on the material [11].

The objective of this work is to develop a material than can deform importantly at very low stress without hysteresis and softening effect and presenting good tear strength. However, it remains difficult to obtain a material having both glassy and rubbery properties [4]. Figure 1 adapted from Bouchereau et al. [12] summarizes our objective and the difficulties to achieve this goal by only controlling the chemistry of the thermoplastic elastomer. Actually, the properties of elastomers vary in function of certain parameters which depend on the vulcanization. It is possible to intercede at several levels in order to alter the crosslinking, for example by varying the vulcanization time. The maximum strain that can be reached by a vulcanized elastomer depends on the average distance between two junctions in the macromolecular network, usually quantified by the crosslinking density. Some mechanical 
characteristics are improved significantly with the crosslinking density such as dynamic or static module. We can understand easily that greater flexibility of the chains and thus high deformability of macroscopic material is observed for a low crosslinking density. However other properties also depend on the degree of crosslinking such as tear strength and fatigue strength, dissipation, hardness. But as these dependencies often develop antagonistically, it is difficult to find an optimal degree of crosslinking compared to the different criteria of behavior.

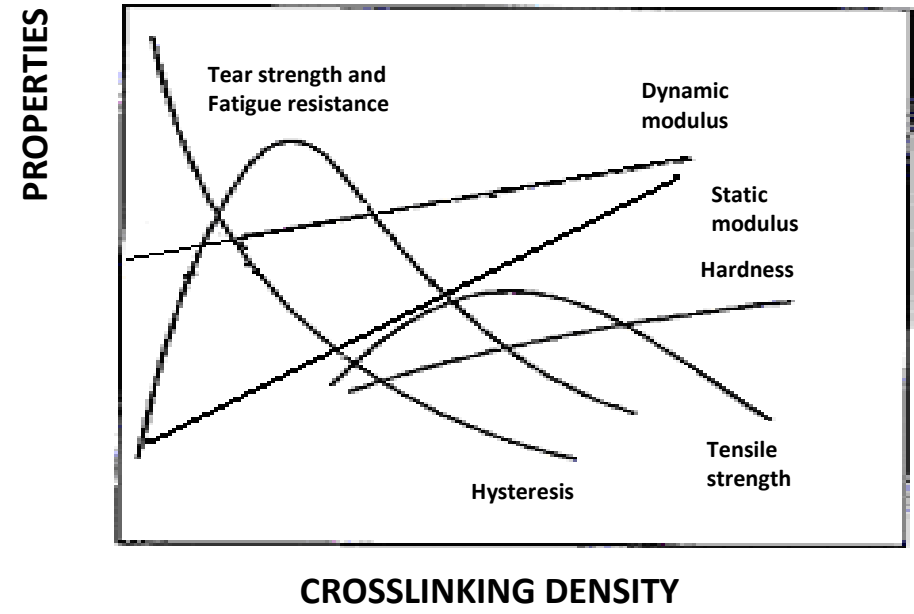

Figure 1: Influence of the cross-linking density on different mechanical properties [12].

Most studies aim at improving the elastic modulus, elongation at break or work of fracture by incorporating nanoparticles to rubber matrices [13-16]. But few of them consist in combining high elasticity, low stress and good tear strength. Little work focuses on the possible combination of high elastic properties, high elongation, low Young's modulus and an improvement of tear strength. Works, based on nanoparticles, enhance the fact that using nanofillers, such as nanoclay, leads to an improvement of tear strength and elastic modulus [17]. Actually, the literature presents the fact that nanoclay can act as an anti-plastic barrier on the material thanks to a combination of chemical and physical interactions with the matrix: the nanoparticles will interact with the linear polymer sequences, leading to an improvement in tear resistance and an extremely large reversible elongation [18-19]. It also reported that 
incorporation of nanoparticles leads to a decrease of elongation at break in case of poor dispersion. This observation is to consider with care for materials under high strain [20-21]. Actually, those fillers act as rigid microdomains and at the end, one has to face the same difficulties as described previously. A solution is to modify the surface of the nanofillers to reach the objective. Recent works show the possibility of improvement of the elongation at break properties of rubbers by the addition of lamellar fillers. Zhenjung et al. studied the influence on the mechanical properties of a styrene-isoprene-butadiene rubber in presence of a suspension of multi-layered organophilic montmorillonite in the toluene or the cyclohexane [22]. They noticed that fillers are better dispersed by the toluene and give greatly dispersed nanocomposites based elastomer. They observed an improvement of the elongation at break of the rubber from 500 to $700 \%$ for an addition of $3 \mathrm{wt} \%$ of montmorillonite in the toluene. Chen-Yang et al. as for them studied the influence of a lamellar nanofiller based organomodified montmorillonite by aminolauric acid (ALA) on the mechanical behavior of a thermoplastic urethane (TPU) increasing elongation at break from 600 to $1400 \%$ [23]. Deng and al worked on a composite system based poly (D, L-lactide) or PDLLA with precipitated $\left(\mathrm{NH}_{4}\right)_{2} \mathrm{HPO}_{4}$ apatite filler modified in a solution of $\mathrm{Ca}\left(\mathrm{NO}_{3}\right)_{2}$ in presence of $\mathrm{SnOct}_{2}$. By using $10 \mathrm{wt} \%$ nanoapatite, the elongation at break of the composite increased from 20 to $1550 \%$ [24].

Finally, the works of Haraguchi and al. highlighted that the incorporation of an important quantity of hydrophilic nanosilica based silsesquioxane, sepiolite, nanoclays, nanotubes of carbons (between 5.5 and $23 \mathrm{wt} \%$ ) in a hydrophobic matrix of poly (2-methoxyethyl acrylate) (PMEA) allowed to obtain very interesting mechanical and optical properties, in particular complete reversibility for deformation up to $3000 \%$ without damages by mechanical necking [17]. 
The present work concerns the new functionalization of sepiolite nanoparticle with thermoplastic elastomer based styrene ethylene butadiene styrene (SEBS). The study will contribute to better understand how the proportion of nanoparticles and the interface treatment influence several mechanical properties by comparison with cast-calendared and dip coating processes.

\section{Materials \& Methods}

\subsection{Materials}

The thermoplastic elastomer used in this study is a SEBS Thermoflex 10H730 from Plastic Technology Service LTD. This triblock copolymer (Figure 2) is a very soft grade (11 Shore A) presenting an important elongation at break and a low modulus.

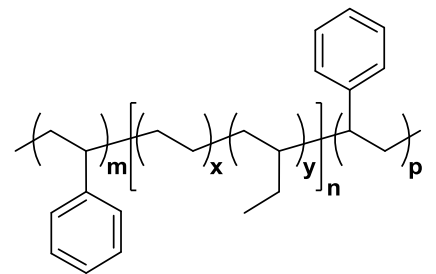

SEBS

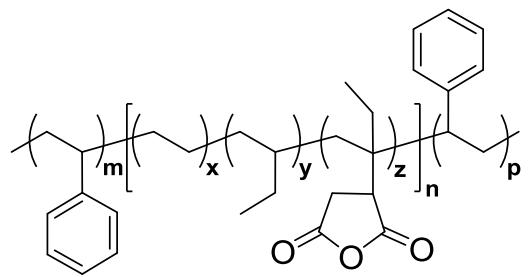

SEBS-g-MA

Figure 2: Chemical structure of SEBS and SEBS-g-MA.

The weight ratio of styrene/ethylene-butadiene is about 5/95, and the weight ratio of ethylene/butadiene in the soft segment is about 70/30. This composition has been determined by comparing the DSC thermogram to literature $[11,25-26]$. The SEBS polymer grafted with maleic anhydride (SEBS-g-MA) FG1901W was supplied by Kraton.

The pristine sepiolite (noted S1 in the text) used was a high purity natural sepiolite Pangel S9 from Tolsa. (3-Aminopropyl)triethoxysilane was supplied by SIGMA-ALDRICH and was used as received. Similarly to palygorskite, the structure of sepiolite is highly porous. This hydrated magnesium silicate $\left(\mathrm{Si}_{12} \mathrm{Mg}_{8} \mathrm{O}_{30}(\mathrm{OH})_{4}\left(\mathrm{H}_{2} \mathrm{O}\right)_{4}, 8 \mathrm{H}_{2} \mathrm{O}\right)$ is based on $\mathrm{SiO}_{4}$ tetrahedra 
layers, with an inversion of the apical ends every six units. These layers are interconnected by $\mathrm{MgO}_{6}$ octahedra, thus creating nanochannels of $3.5 \times 10.6 \AA^{2}$ in cross-section [27]. Two types of water molecules are present in the structure: water coordinated to $\mathrm{Mg}^{2+}$ ions at the edges of the octahedral layers $\left(\mathrm{H}_{2} \mathrm{O}_{\text {coord }}\right)$ and zeolithic water in the channels $\left(\mathrm{H}_{2} \mathrm{O}_{\mathrm{zeol}}\right)$, hydrogenbonded to coordinated water molecules.

\subsection{Functionalization of sepiolite}

To improve mechanical performances of sepiolite nanofibers /SEBS films, a new strategy for sepiolite functionalization was developed. This strategy based on a two-step reaction is presented by the Figure 3. The first step is the sepiolite treatment by APTS to introduce amine groups at the filler surface. This silylation reaction is not carried in anhydrous condition and the auto-condensation of the silanes units leads to the formation of a multilayer grafting. The second step is the functionalization of the sepiolite nanofibers with SEBS chains by reaction between the amine groups and anhydride maleic groups of a SEBS-g-MA copolymer. This reaction leads to the formation of an amide group and so to a covalent bond between the sepiolite surface and the SEBS chains.

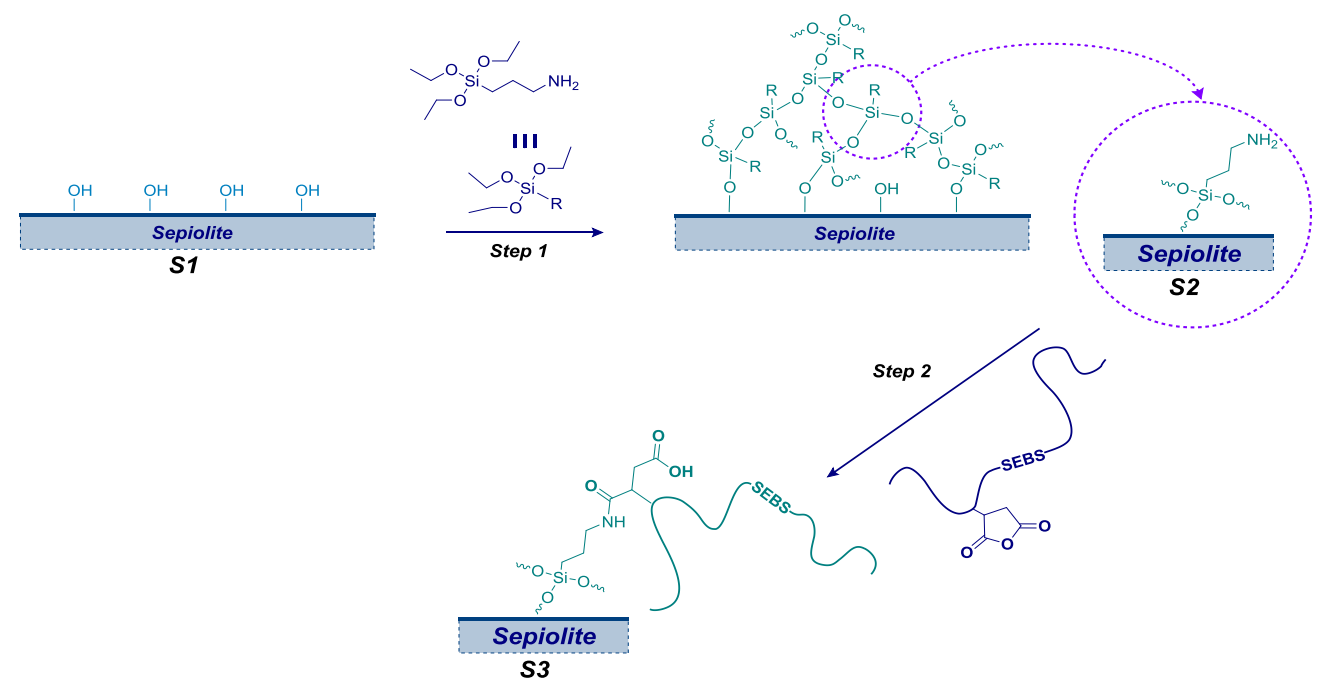

Figure 3: Functionalization of sepiolite nanofibers by a 2 steps procedure. 


\subsubsection{Functionalization of sepiolite by amino groups (S2)}

Into a $250 \mathrm{ml}$ flask fitted with a condenser were introduced $10 \mathrm{~g}$ of sepiolite Pangel S9 (S1), $1 \mathrm{~g}\left(4.5 \times 10^{-3} \mathrm{~mol}\right)$ of (3-aminopropyl)triethoxysilane (APTES) and $100 \mathrm{ml}$ of an ethanol/water (90/10) solution. The mixture was then stirred and heated at solvent reflux for 15 hours. The mixture was next centrifuged (speed: $5000 \mathrm{rpm}$ ) to eliminate the liquid phase and washed three times with acetone. Finally, the obtained sepiolite S2 was dried under vacuum before characterization.

\subsubsection{Functionalization of sepiolite by SEBS polymer chains (S3)}

Into a $250 \mathrm{ml}$ flask fitted with a condenser were introduced $10 \mathrm{~g}$ of filler, $1 \mathrm{~g}$ of SEBS-g-MA and $100 \mathrm{ml}$ of toluene. The mixture was then stirred and heated at solvent reflux for 15 hours. The mixture was next centrifuged (speed: $5000 \mathrm{rpm}$ ) to eliminate the liquid phase and washed two times with toluene and acetone. Finally, the filler was dried under vacuum before characterization.

\subsection{Preparation of the SEBS formulations for the dip coating process}

In a first step the SEBS elastomer was dissolved in toluene under vigorous stirring. When the polymer dissolution is complete, sepiolite (S1, S2 or S3) and SEBS-g-MA can be added. The different formulations tested in this study are summarized in Table 1. 
Table 1: Composition of the different formulations used for the dip coating process.

\begin{tabular}{|l|c|c|c|c|}
\hline Formulation & $\begin{array}{c}\text { SEBS Thermoflex } \\
\text { 10H730 } \\
(w t \%)\end{array}$ & $\begin{array}{c}\text { Sepiolite S1, } \\
\text { S2 or S3 } \\
(w t \%)\end{array}$ & $\begin{array}{c}\text { SEBS-g-MA } \\
\text { Kraton FG1901X } \\
(w t \%)\end{array}$ & Toluene \\
\hline SEBS & 15 & - & - & 85 \\
\hline SEBS/S1 & 14.85 & 0.15 & - & 85 \\
\hline SEBS/S2 & 14.85 & 0.15 & - & 85 \\
\hline SEBS/S2/SEBS-g-MA & 14.85 & 0.14 & 0.01 & 85 \\
\hline SEBS/S3 & 14.85 & 0.15 & - & 85 \\
\hline
\end{tabular}

2.4 Dip coating process

\subsubsection{Description of the dip-coating apparatus}

The machine allows preparing SEBS films from the different formulation prepared (Table 1). The process can be divided in three different steps (Figure 4). In a first time, the glass pipe is dipped in the SEBS formulation with a controlled speed. Then, when the lower limit position has been reached, the tube is removed at controlled speed and acceleration. Finally, the tube is put in a horizontal position and rotated during a given time to dry the SEBS film. The different parameters of these three steps define a cycle. The type and number of the cycles affect the quality of the obtained elastomer films.

The dip-coating machine is composed of several electric motors and sensors. The global process is the combination of different operations and for each a motor (Servo HITEC HS$805 \mathrm{MG}$ with a torque of $24.7 \mathrm{~kg} / \mathrm{cm}$ for $6.0 \mathrm{~V}, 25 \mathrm{rpm}$ and maximal speed of $2 \mathrm{~cm} / \mathrm{s}$ ) using a PIC microcontroller (Microship) is used to obtain a specific displacement. The first one is a DC motor which drives a ball screw to obtain a vertical displacement of the tube. The second one is a servomotor which allows the tube to switch from a horizontal to a vertical position. The third one is a DC motor which allows the rotation of the tube via a pulley-belt system. The different sensors (Honeywell) allow the control of the vertical movement and the horizontal position of the tube. 


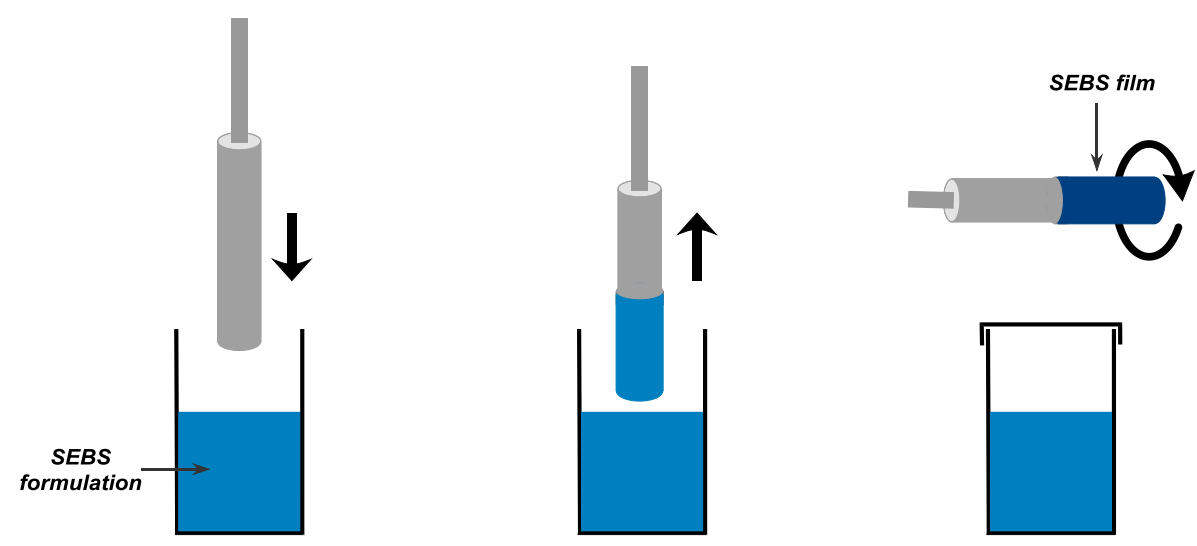

Figure 4: Dip coating procedure for the formation of the SEBS films.

To obtain a SEBS film, the apparatus is programed with values of rate of descent, rate and deceleration of ascent, duration for the dry step and number of cycles.

As a comparison, SEBS was also cast-calendared with a laboratory-scale extruder Polylab system composed of a HAAKE RheoDrive4 motor coupled with a HAAKE Rheomex 19/25 OS single screw extruder with a Maddock mixer equipped with a flat die. Films of 0.4 mm thick are obtained.

\subsubsection{Effect of acceleration on the thickness of the SEBS films}

The first tests showed that the obtained films did not present the same thickness throughout their length. To reduce these differences of length different values for the deceleration parameter were tested. Figure 5 shows the influence of deceleration value on the thickness gradient for the obtained film.

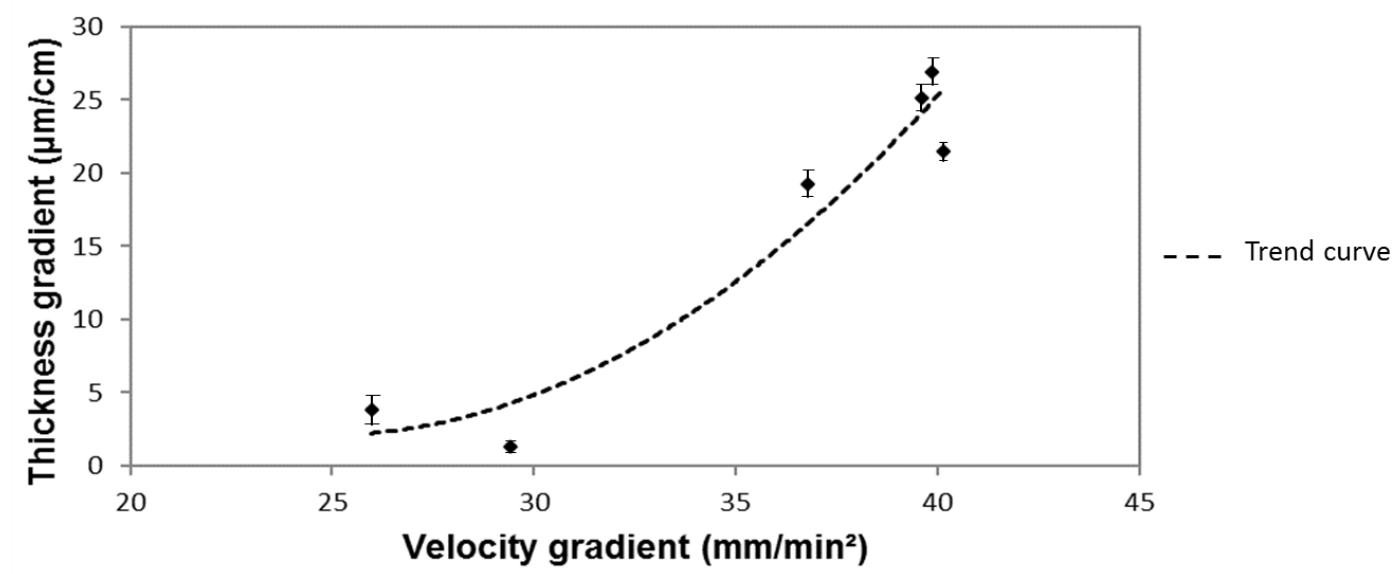


Figure 5: Thickness gradient $(\mu \mathrm{m} / \mathrm{cm})$ as a function of the velocity gradient $\left(\mathrm{mm} / \mathrm{min}^{2}\right)$ for a SEBS/S1 formulation.

The lower thickness gradients were obtained with values of deceleration between $25 \mathrm{~mm} / \mathrm{min}^{2}$ and $30 \mathrm{~mm} / \mathrm{min}^{2}$. These velocity gradients correspond to values of thickness gradient about $5 \mu \mathrm{m} / \mathrm{cm}$.

\subsubsection{Number of cycles}

For a given formulation the number of cycles determines the thickness of the obtained film. The measures of thickness were obtained with a Micrometer and the uncertainties were estimated on 5 samples. The formulations used for this study give a layer with a thickness about $100 \mu \mathrm{m}$ for each cycle. For this study, the number of cycles was fixed at 5 for an overall thickness of $500 \pm 50 \mu \mathrm{m}$ for the obtained films.

\subsubsection{Effect of the concentration of the SEBS formulation on the thickness of the obtained films}

The SEBS concentration of the formulation used for the dip coating process is directly related to the thickness on the obtained film. Figure 6 presents a linear evolution of the film thickness in function of the SEBS concentration.

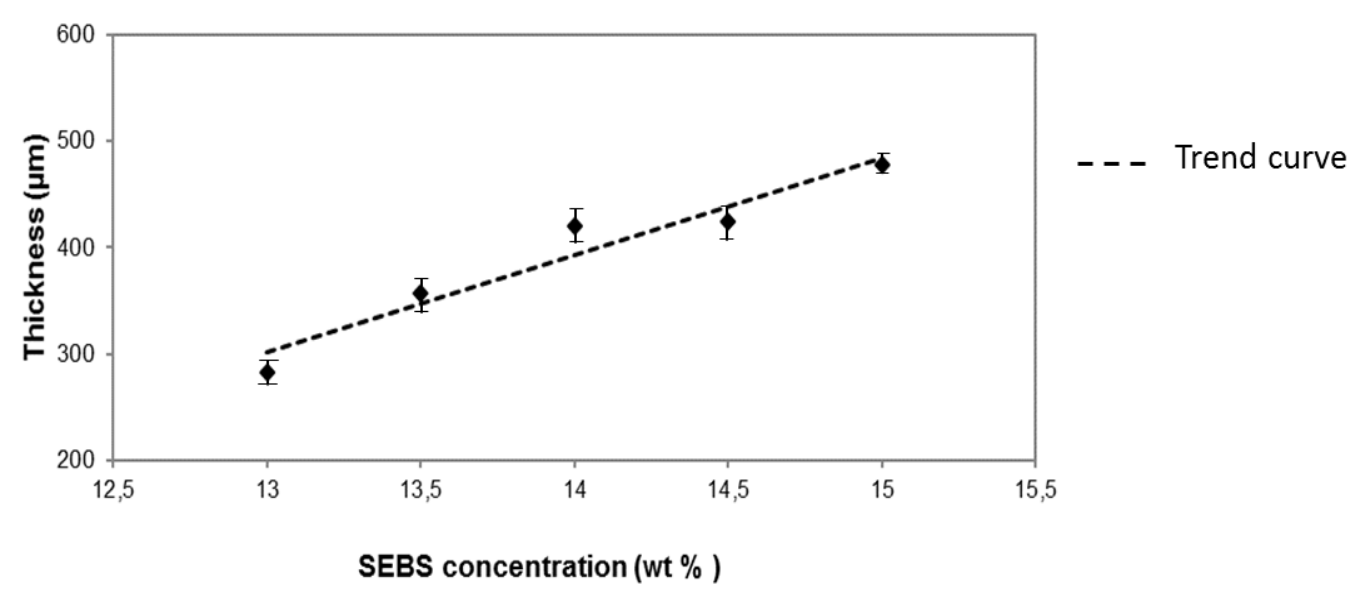

Figure 6: Thickness of the obtained film as a function of the SEBS concentration. 


\subsection{Characterizations}

\subsubsection{Thermal characterization}

Thermal characterization was carried out by thermogravimetric analysis (Perkin Elmer Pyris1 instrument) on $10 \mathrm{mg}$ of samples, under nitrogen. Samples were first heated at $10^{\circ} \mathrm{C} / \mathrm{min}$ from 25 to $110^{\circ} \mathrm{C}$, followed by an isotherm at $110^{\circ} \mathrm{C}$ for $10 \mathrm{~min}$, in order to evacuate all adsorbed water molecules. They were then heated again from 110 to $900^{\circ} \mathrm{C}$, at $10^{\circ} \mathrm{C} / \mathrm{min}$, in order to eliminate the grafted groups.

\subsubsection{FTIR characterization}

IR spectra were measured using a Bruker IFS66-IR Spectrometer at room temperature, where 32 scans at a resolution of $4 \mathrm{~cm}^{-1}$ were signal averaged.

\subsubsection{XRD characterization}

Morphologies were analyzed using a Bruker D8 diffractometer using CuKa radiation. Data were collected between 2 and $33^{\circ}$ by step of $0.02^{\circ}$ using an X-ray generator with $\lambda=0.15406$ $\mathrm{nm}$ and at $40 \mathrm{kV}$ operating voltage and $20 \mathrm{~mA}$ current.

\subsubsection{TEM characterization}

The morphology of composites was examined by transmission electron microscopy (TEM). Samples were cut using a LEICA UC7 ultramicrotome at $-156^{\circ} \mathrm{C}$. Sections of approximately $70 \mathrm{~nm}$ were observed with a PHILIPS CM 120 TEM at $80 \mathrm{kV}$.

\subsubsection{Mechanical Properties}

Cyclic tensile tests and monotonic tear strength tests were performed at $23^{\circ} \mathrm{C}$ on a ZWICK TH010 universal testing machine with a load cell capacity of $500 \mathrm{~N}$ at a speed of 500 $\mathrm{mm} / \mathrm{min}$

Five specimens used for tensile and tear analyses were cut from the films obtained by the dip coating process (Figure 6). Note that for the tear strength samples, the dimensions given by the French standard (NF EN 12310) [28] were divided by two because of the sizes of the films 
produced by the dip coating procedure. Tensile samples were submitted to successive load / unload cycles at an elongation of $300 \%, 450 \%$ and $600 \%$ (8 cycles in each cases), and a final load up to fracture (Figures 7 and 8). Stress at the first load at $600 \%$ is evaluated, as well as the stress and elongation at break. The elastic modulus is taken as the slope of initial tangent to the stress/strain curve at the first load.
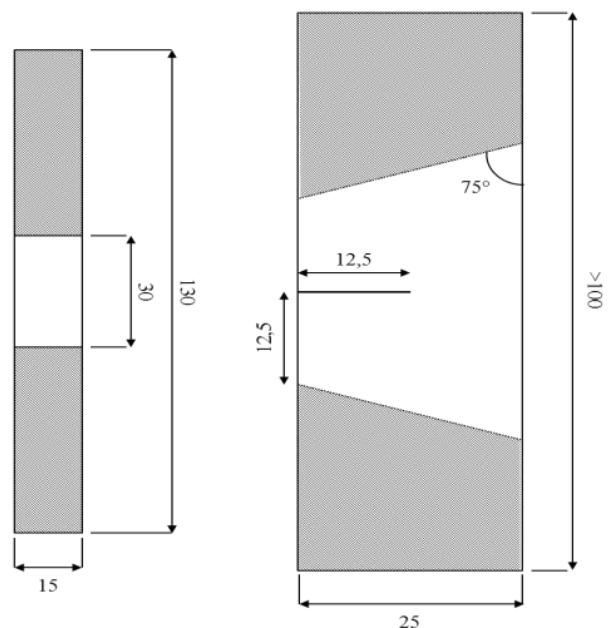

Figure 7: Shape of the samples used for (left) the cyclic tensile and (right) tear strength tests (values in $\mathbf{m m}$, grey areas are placed between clamps).

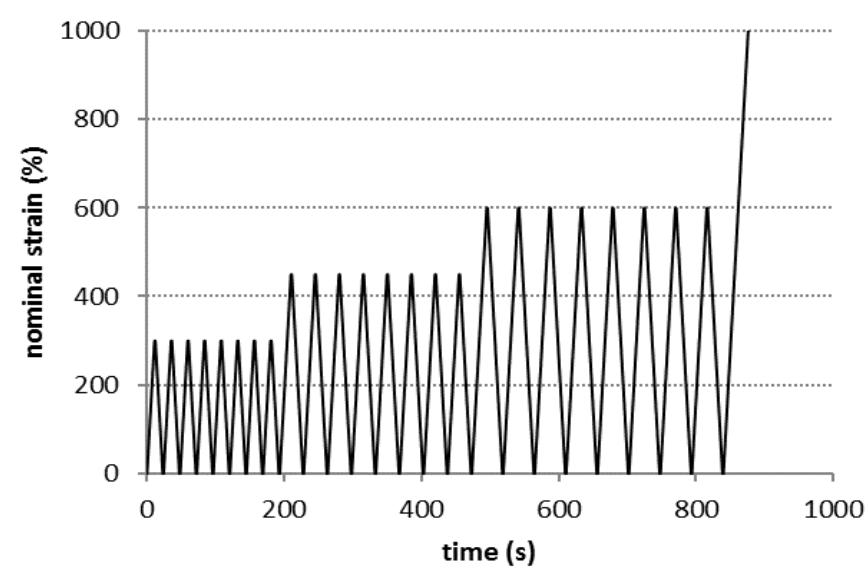

Figure 8: Loading program for cyclic tensile tests.

Let $W_{i}$ be the elastic strain energy density of the $i^{\text {th }}$ load, and $\mathrm{H}_{8}$ the elastic strain energy released after the last unload (Figure 9). A relative stabilization ratio (SR) is calculated using Equation 1 and characterizes the Mullins effect. A low value of SR corresponds to a low stress softening. A relative viscoelastic ratio (VR) is also determined from the last cycle using 
Equation 2 and quantifies the viscoelasticity of the stabilized material. Ideally, a low (VR) ensures a quasi-elastic behavior of the material.

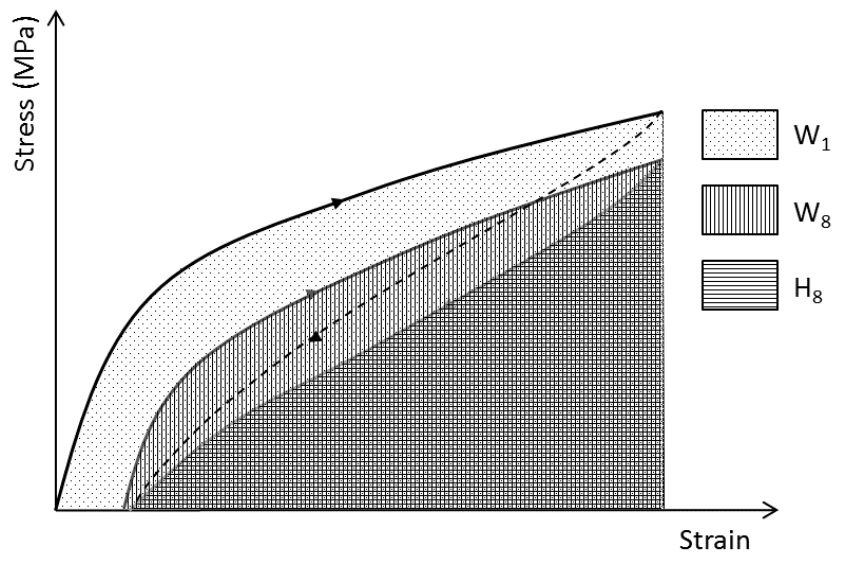

Figure 9: Elastic strain energy densities stored and released during load/unload cycles of elastomeric material.

Equation 1:SR $=\frac{W_{1}-W_{g}}{W_{1}} * 100$

Equation $2: V R=\frac{W_{\Omega}-H_{g}}{W_{g}} * 100$

The tear strength (T) as defined in the French standard NF EN 12310-2 and the dissipated surface energy to initiate and propagate the crack $\left(E_{d i s}\right)$ are also evaluated for all specimens (Equation 3 and Equation 4). These parameters are obtained by dividing respectively the maximal strength $\left(\mathrm{F}_{\max }\right)$ by the thickness $(e)$ and the dissipated energy $\left(\mathrm{W}_{\text {dis }}\right)$ by the cross section $(S)$, which is the area of the resistive ligament of the tear strength samples (Figure 7), in order to compare the samples (Figure 10).

Equation $3: T=\frac{F_{\max }}{e}$

Equation $4: E_{\text {dis }}=\frac{w_{\text {dis }}}{s}$ 


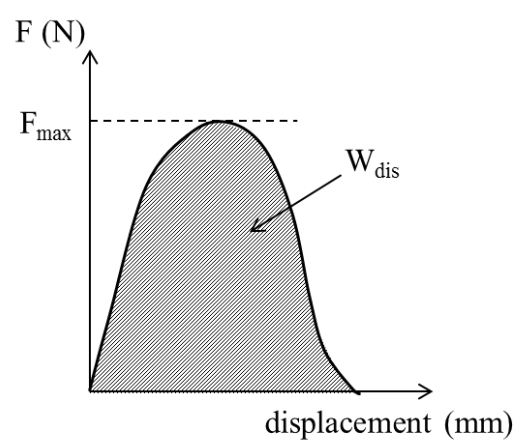

Figure 10: Tear strength and surface dissipated energy.

\section{Results and discussions}

\subsection{Surface modification of sepiolite nanofibers}

In order to check to what extent sepiolite has been modified, S1, S2 and S3 samples have been characterized by TGA and IR spectroscopy.

As detailed by several authors [29-30], the heating of pristine sepiolite reveals a multi-step dehydration process, corresponding to the loss of zeolitic water first and then of coordinated water. The zeolitic water is lost in one step, between room temperature and $100^{\circ} \mathrm{C}$, while the coordinated water is lost in two steps, between 100 and $300^{\circ} \mathrm{C}$ first and then between 300 and $600^{\circ} \mathrm{C}$. Another step is then observed, from $800^{\circ} \mathrm{C}$, corresponding to the dehydroxylation of sepiolite anhydride which loses its structure, resulting in the formation of enstatite and silica. Thermal characterization of pristine sepiolite and both modified sepiolites was carried out by TGA, following the protocol described in the experimental section. The isotherm step at $110^{\circ} \mathrm{C}$ was used to dry samples so as to avoid any disturbance of the results by the weight loss due to the zeolitic water (Figure 11a). During this step, pristine sepiolite undergoes a $6 \%$ weight loss (zeolitic water loss). In the case of organo-modified sepiolites the weight loss is lower compared to pristine sepiolite: 4.1 and $2 \%$, respectively. This can be explained by (i) a lower amount of zeolitic water in the organo-modified sepiolite and/or (ii) the increase of the molecular weight of modified sepiolite due to the presence of organic molecules. Considering 
that all the samples are in the same hydration state after the first step (loss of the zeolitic water), the comparison of their behavior between 110 and $900^{\circ} \mathrm{C}$ (Figure 11b) allows checking to what extent the sepiolites have been modified. The first two steps observed, between 110 and $800{ }^{\circ} \mathrm{C}$ on Figure $11 \mathrm{~b}$, for pristine sepiolite (straight line) corresponds to a weight loss of $7.4 \mathrm{wt} \%$. They can be attributed to the loss of coordinated water (dehydration). Then, after $800{ }^{\circ} \mathrm{C}$, dehydroxylation occurs. Between 110 and $800{ }^{\circ} \mathrm{C}$, the modified sepiolites show significantly different behaviors from that of pristine sepiolite but also one from the other. Their behavior is however similar to that of pristine sepiolite above $800{ }^{\circ} \mathrm{C}$, probably corresponding also only to dehydroxylation. Between 110 and $800^{\circ} \mathrm{C}$ the total weight loss of S2 and S3 samples is respectively 10.2 and $18.4 \mathrm{wt} \%$, i.e., 2.8 and $11 \mathrm{wt} \%$ more than that of pristine sepiolite. This is a first indication that the sepiolites have been modified. Furthermore, the more significant weight loss for S3 occurs between 380 and $400^{\circ} \mathrm{C}$ corresponding to the same thermal degradation than pure SEBS-g-MA (Figure 11b). In order to prove the grafting of the SEBS chains at the surface of sepiolite, the S3 has been analyzed by FTIR spectroscopy and its spectra compared to that of SEBS and SEBS-g-MA polymers (Figure 12). The modified sepiolite spectrum shows the presence of a signal between 2830 and $2990 \mathrm{~cm}^{-1}$ (Figure 12a) characteristic of the SEBS chains which can be observed for the spectra of SEBS and SEBS-g-MA. A signal centered at $1660 \mathrm{~cm}^{-1}$ can also be observed for the S3 sample (Figure 12b) and was attributed to the amide and imide functions formed after reaction between the amine functions of the S2 sample and the anhydride functions of the SEBS-g-MA chains. This signal which does not appear for the SEBS and SEBS-g-MA polymers proves the grafting of the SEBS chains by the formation of a covalent bond. 

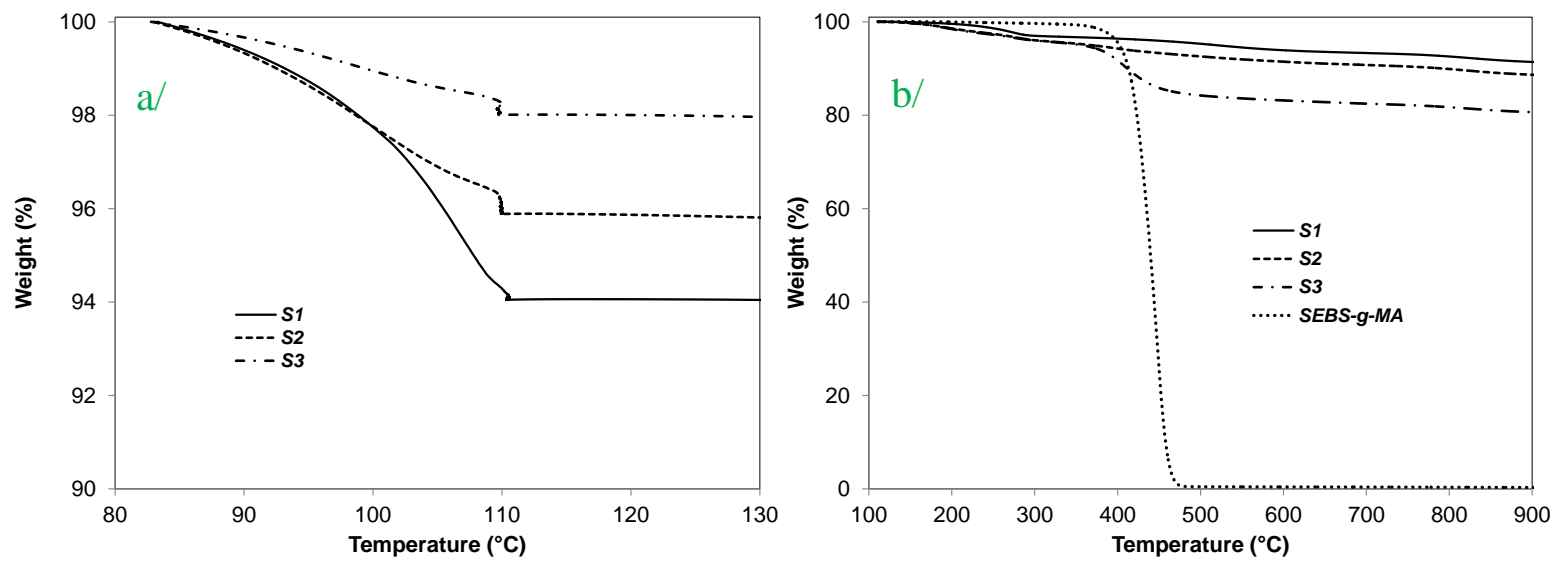

Figure 11: TGA under nitrogen of S1, S2 and S3 samples, (a) from 25 to $110^{\circ} \mathrm{C}$ at $10^{\circ} \mathrm{C} / \mathrm{min}$ followed by an isotherm at $110^{\circ} \mathrm{C}$ for $10 \mathrm{~min}$, (b) from 110 to $900^{\circ} \mathrm{C}$ at $10^{\circ} \mathrm{C} / \mathrm{min}$.
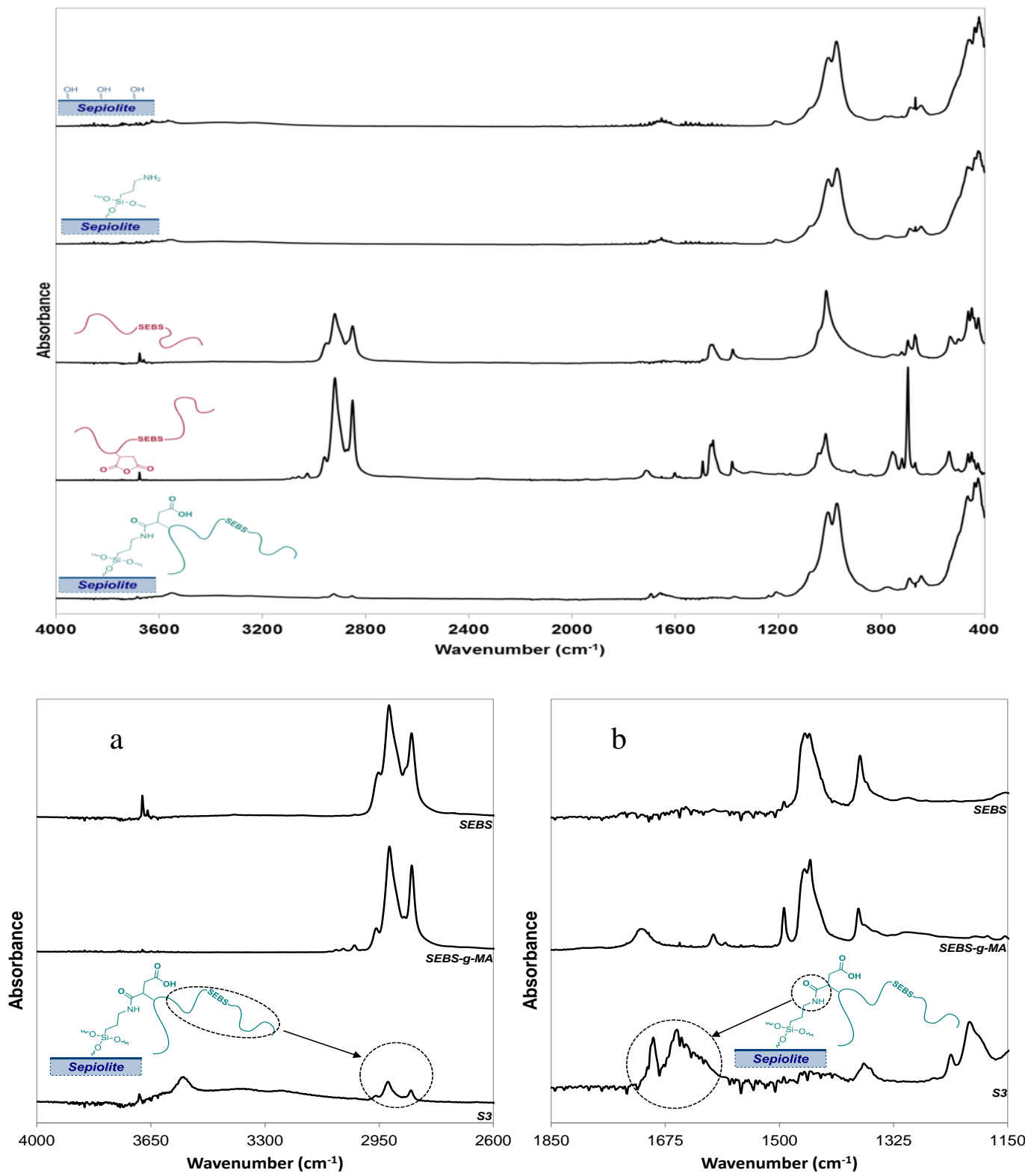

Figure 12: ATR-FTIR spectra of SEBS, SEBS-g-MA and sepiolite/APTES/SEBS-g-MA (S3) samples. 


\subsection{Characterization of the nanocomposites}

\subsubsection{WAXD studies in low angle range}

Nanocomposites were analyzed by XRD in order to estimate the extent of clay dispersion in the SEBS matrix. XRD patterns for nanocomposites filled with $1 \mathrm{wt} \%$ of neat and different modified sepiolite grafted SEBS into SEBS matrix (S1, S2, S3) in comparison with pristine sepiolite are showed Figure 13.

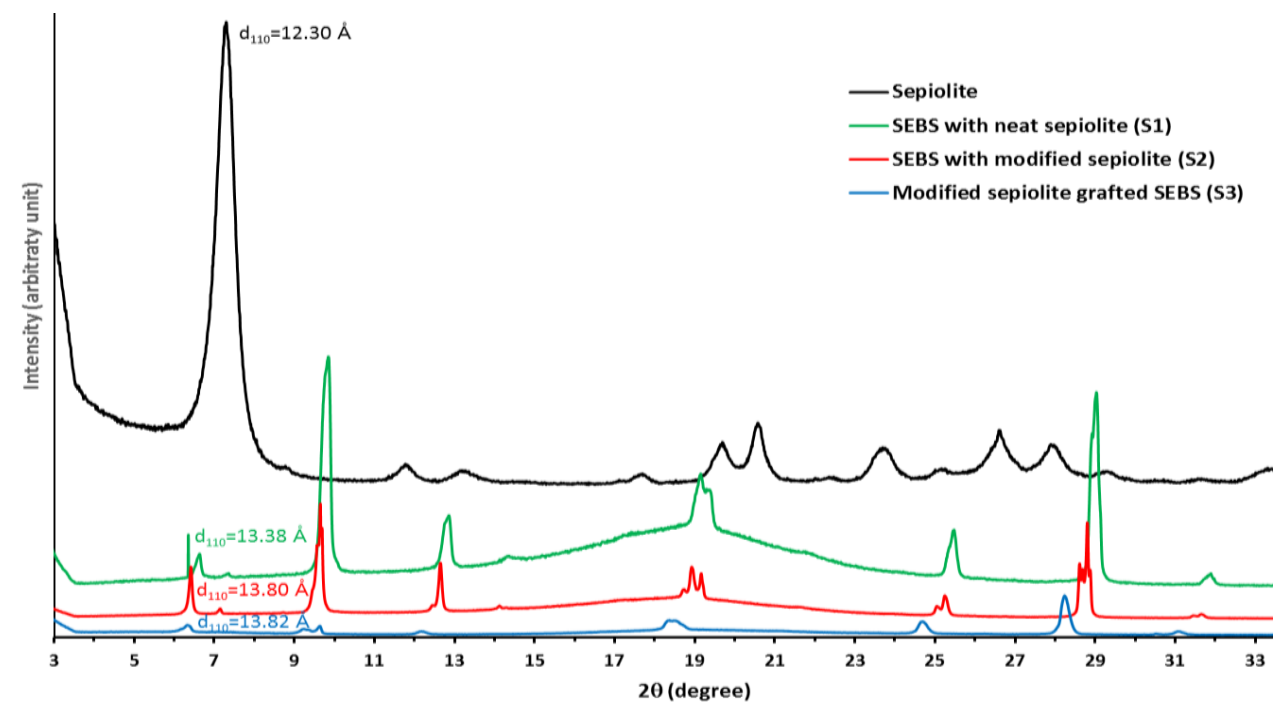

Figure 13: X-ray scattering patterns of neat sepiolite (S1) and modified sepiolite non-grafted (S2) and grafted SEBS (S3) into SEBS matrix with respect to pristine sepiolite.

The interlayer distance $d_{110}$ for the pristine clay is $12.20-12.40 \AA$ [31] for a peak in the range of 7.2-7.4 ${ }^{\circ}(2 \theta)$. In contrast, dispersed hybrids exhibit almost no distinct features in this $2 \theta$ range except a left displacement of this 110 peak from $7.26^{\circ}$ to $6.39^{\circ}$ corresponding to an interlayer distance of $13.82 \AA$ (S3). Despite this shiftless, in the present case, a very low definite diffraction pattern is an evidence for high degree of dispersion of sepiolite in the matrix when modified sepiolite is carried out. Actually, the fact to chemically organo-modify sepiolite (S2) then to graft S2 with $\mathrm{SEBS}_{\text {gma }}(\mathrm{S} 3)$ highlights this decrease of the definite diffraction pattern. This is in well accordance with the observation made by Benlikaya et al. and by Roy et al. [32-33]. Unlike other clay based-montmorillonite (MMT) [34], where 
individual tetrahedral and octahedral layers can easily be separated, sepiolite exhibits a structure where individual TOT (tetrahedral octahedral tetrahedral) layers are strongly bonded. Unlike smectite clays, here fiber bundles or aggregates get separated into nanometer dimension when these fillers are dispersed into the polymer matrix. Relative dispersion of the clay refers to a good isolation of the individual fibers from each other. As shown in the Figure 13, almost disappearance of the 110 peak of sepiolite in XRD patterns of nanocomposites is considered as an evidence for good dispersion of the main fraction of sepiolite fibers within SEBS matrix. In spite of our best efforts by various techniques, the layers in sepiolite could not be separated earlier. But in the case of in situ synthesis of nanocomposites, greater extent of dispersion is achieved.

\subsubsection{TEM studies}

A reasonably good dispersion of neat sepiolite is observed in SEBS (Figure 14a), with few clusters of higher density of particles (dark phases) and aggregates of size $0.2-0.5 \mu \mathrm{m}$ (dark needles). In SEBS/S3 sample, bigger aggregates $(1 \mu \mathrm{m})$ can be observed, while the dispersion of sepiolite in clusters is good (Figure 14b). Clusters are fewer in number but larger in size. The surface modification of sepiolite seems to be in favour of this clustering effect and this is confirmed by the XRD decrease of peak intensity showing a more important amorphous/crystalline sepiolite ratio. Regardless of these clusters, a homogeneous zone is indicative of a good dispersion of sepiolite.

To the authors, such phenomenon has not been reported yet, but could be explained by a competition of affinity between the SEBS grafted molecule and the SEBS matrix which should lead to a good dispersion of sepiolite or another SEBS grafted molecule which lead to a clustering effect. 


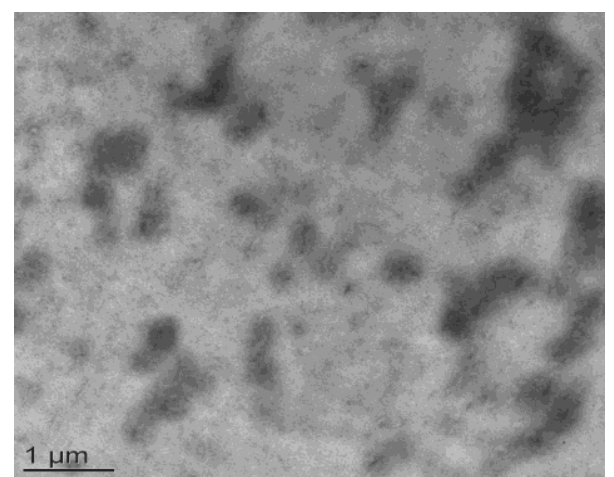

a) $\mathrm{S1}$

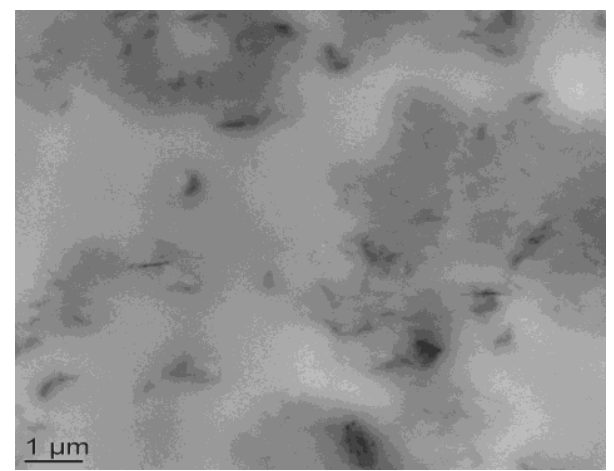

b) S3

Figure 14: TEM images of neat sepiolite (S1) and SEBS grafted sepiolite (S3) into SEBS matrix.

\subsection{Mechanical properties}

Figure 15 and Figure 16 display the shape of typical stress/strain curve and tear strength/displacement curve respectively. All the data are gathered in Table 2 and Table 3. Concerning the processing, casted and dip-coated SEBS properties can be compared. It appears that dip-coating provides SEBS the ability to be more deformable and flexible at a low stress with a moderate Mullins effect and viscous behavior. Moreover, the resistance to tear of the dip-coated SEBS is greatly improved compared with the casted SEBS.

If we compare the formulations obtained by dip-coating process, it can be noted that the incorporation of untreated sepiolite (S1) contributes to an improvement of the tear strength and the dissipated energy but also to an increase of the Mullins effect and a decrease of the ability of the elastomer to deform. The introduction of amine groups on sepiolite surface (S2) without additional interfacial agent slightly improves the resistance to tear of the material, from $3.3 \mathrm{~N} / \mathrm{mm}$ (pristine SEBS) to 3.7 (S2) but the mechanical properties, as stress at $600 \%$, ultimate stress and strain, are still degraded. 


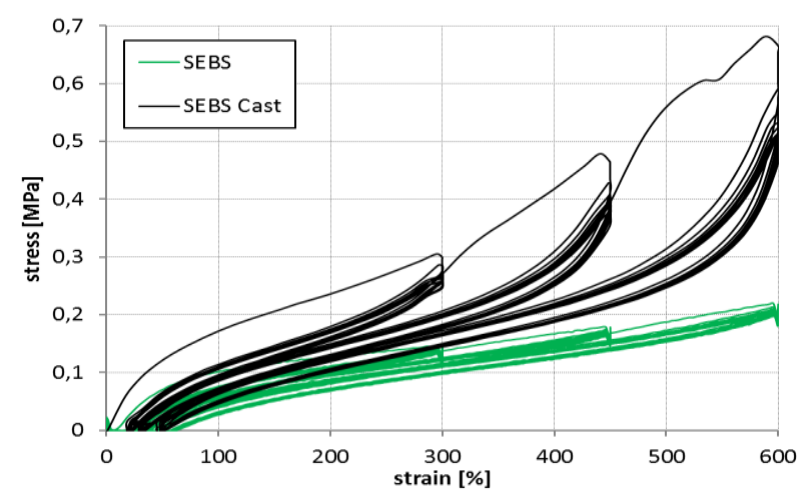

Figure 15: Typical stress/strain curves for tensile cyclic loading.

Adding SEBS-g-MA to S2 sepiolite allows preserving the initial behavior of SEBS (no significant differences in VR, SR, ultimate stress and strain values) while significantly improving tear strength and energy dissipation. As expected an important increase in resistance to tear is observed for SEBS with grafted sepiolite (S3), but sadly it is also responsible for an important increase in the Mullins effect. The increase in ultimate stress (crystallization phenomenon) is not an issue since the stresse at $600 \%$ is not impacted. However, observed improvements with grafted sepiolite could be further improved by considering a better dispersion of sepiolite in the matrix. The elastic modulus is not strongly impacted by the filler or its treatment.

Table 2 : Values of VR, SR, stress at $\varepsilon=600 \%$, and ultimate stress and strain for the SEBS formulations.

\begin{tabular}{|l|l|l|l|l|l|l|}
\hline & SR $(\%)$ & VR $(\%)$ & $\begin{array}{l}\text { Stress 600\% } \\
(\mathbf{M P a})\end{array}$ & $\begin{array}{l}\text { Ultimate } \\
\text { stress }(\mathrm{MPa})\end{array}$ & $\begin{array}{l}\text { Ultimate } \\
\text { strain (\%) }\end{array}$ & $\begin{array}{l}\text { Elastic modulus } \\
(\mathrm{MPa})\end{array}$ \\
\hline SEBS & $25.9 \pm 1.5$ & $11.5 \pm 0.9$ & $0.19 \pm 0.02$ & $2.6 \pm 0.2$ & $2060 \pm 30$ & $0.27 \pm 0.01$ \\
\hline SEBS/S1 & $33.1 \pm 0.6$ & $9.9 \pm 0.2$ & $0.19 \pm 0.01$ & $2.0 \pm 0.5$ & $1940 \pm 80$ & $0.29 \pm 0.03$ \\
\hline SEBS/S2 & $37.2 \pm 0.2$ & $11.2 \pm 0.1$ & $0.17 \pm 0.01$ & $2.1 \pm 0.3$ & $1920 \pm 40$ & $0.27 \pm 0.02$ \\
\hline SEBS/S2/SEBS-g-MA & $28.2 \pm 0.3$ & $11.4 \pm 0.1$ & $0.18 \pm 0.04$ & $2.3 \pm 0.5$ & $1890 \pm 90$ & $0.29 \pm 0.04$ \\
\hline SEBS/S3 & $39.1 \pm 1.0$ & $6.8 \pm 1.0$ & $0.17 \pm 0.01$ & $2.9 \pm 0.1$ & $1980 \pm 60$ & $0.28 \pm 0.02$ \\
\hline SEBS cast & $49.9 \pm 0.8$ & $14.2 \pm 0.3$ & $0.68 \pm 0.01$ & $3.4 \pm 0.2$ & $1630 \pm 40$ & $0.45 \pm 0.02$ \\
\hline
\end{tabular}




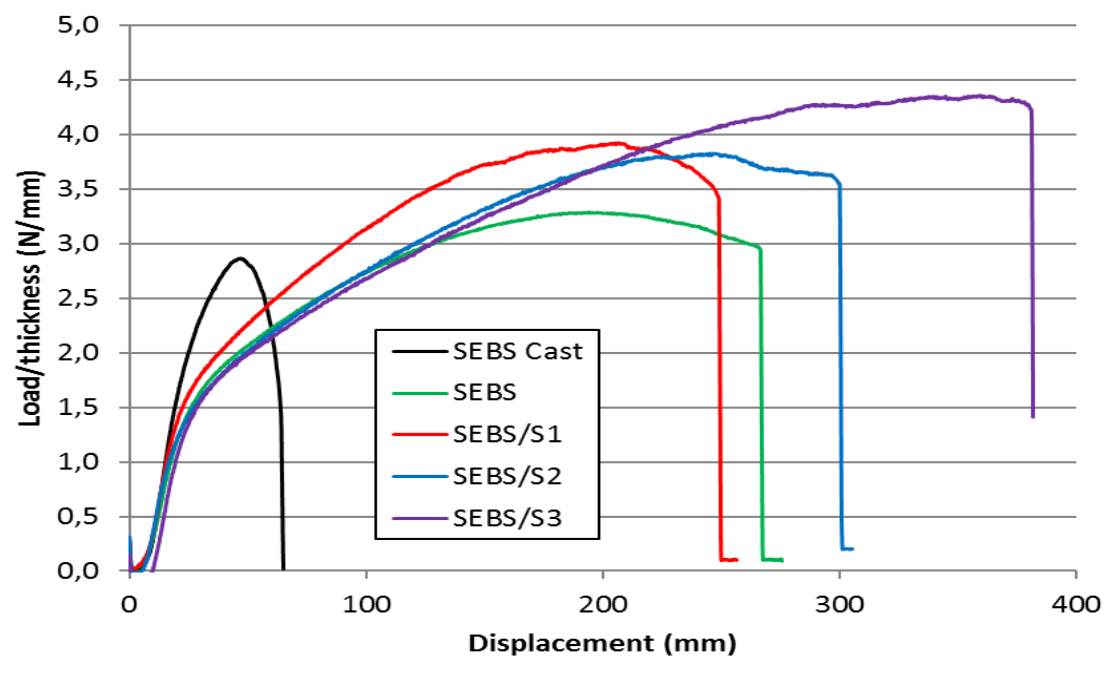

Figure 16: Typical tear strength/displacement curves for SEBS formulations.

Table 3 : Values of tear strength and dissipated energy for the SEBS formulations.

\begin{tabular}{|c|c|c|}
\hline & Tear strength $(\mathrm{N} / \mathrm{mm})$ & $\begin{array}{l}\text { Dissipated energy } \\
\left(\mathrm{mJ} / \mathrm{mm}^{2}\right)\end{array}$ \\
\hline SEBS & $3.3 \pm 0.1$ & $66 \pm 1$ \\
\hline$S E B S / S 1$ & $4.1 \pm 0.5$ & $69 \pm 10$ \\
\hline SEBS/S2 & $3.7 \pm 0.2$ & $70 \pm 5$ \\
\hline$S E B S / S 2 / S E B S-g-M A$ & $4.0 \pm 0.1$ & $84 \pm 3$ \\
\hline$S E B S / S 3$ & $4.2 \pm 0.2$ & $93 \pm 7$ \\
\hline SEBS cast & $2.9 \pm 0.1$ & $10 \pm 2$ \\
\hline
\end{tabular}

The whole results are summarized Figure 17. However, it is important to note that these improvements attributed to the functionalization process of the sepiolite can be further improved by a better dispersion of sepiolite in the matrix. 


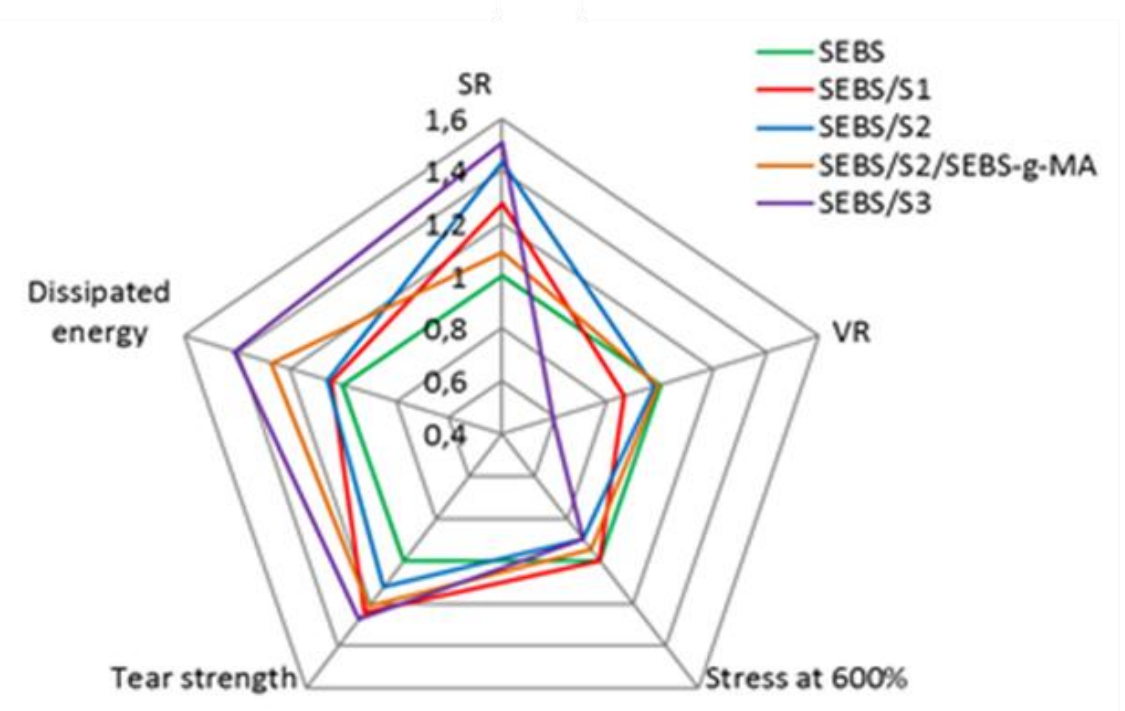

Figure 17: Summary of mechanical results for different formulations.

\section{Conclusion}

An ultrahigh tear resistance and elongation rate of the SEBS-matrix nanocomposite elastomer with ultra-fine acicular sepiolite nanoparticles as fillers were found in the present study by a dip-coating process. The improvement of these performances is not made to the detriment of the flexibility and of the elongation at break of the material which remained constant. The incorporation of functionalized nanoclay improves the dissipated energy during a tear test by $40 \%$, without impacting significantly the stabilization and viscosity rates of pure SEBS. This strong increase is the result of a new process of stoichiometric compatibilisation of the sepiolite with the agent of interface (SEBS-g-MA) then the incorporation of the functionalized nanoparticles within the matrix SEBS. The linear chains of SEBS matrix material were cross-linked through ultra-high surface energy of the acicular sepiolite nanoparticles. These crosslinked SEBS structures resulted in the high fixation strength between SEBS molecular chains and nano-sepiolite particles by a larger number of additional cross-link junctions; it led to a quasi-identical elastic and plastic deformation behavior of the composites when comparing with pure SEBS polymer. 


\section{Acknowledgment:}

This work was financially supported by the AREVA MELOX (N. Lantheaume) and PIERCAN SAS (D. Guérin) companies. TEM samples preparation and observation have been performed at the Centre Technologique des Microstructures, University of Claude Bernard, Lyon 1, France. The authors also thank P. Hangouët, N. Page, G. Chantereau, V. Diaz, T. Dutto and V.B. Nguyen who worked on this project.

\section{References:}

[1] G. Matzeu, A. Pucci, S. Savi, M. Romanelli, F. Di Francesco, A temperature sensor based on a MWCNT/SEBS nanocomposite, Sensors and Actuators A 178 (2012), pp. 94-99.

[2] D. Juárez, S. Ferrand, O. Fenollar, V. Fombuena, R. Balart, Improvement of thermal inertia of styrene-ethylene/butylene-styrene (SEBS) polymers by addition of microencapsulated phase change materials (PCMs) (2011), European Polymer Journal, Volume 47, Issue 2, Pages 153-161.

[3] Hengchong Shi, Dean Shi, Cong Li, Shifang Luan, Jinghua Yin, Robert K.Y. Li, Preparation of functionalized graphene/SEBS-g-MAH nanocomposites and improvement of its electrical, mechanical properties, Materials Letters 133 (2014), pp. 200-203.

[4] C.P. Buckley, C. Prisacariu, C. Martin. Elasticity and inelasticity of thermoplastic polyurethane elastomers: Sensitivity to chemical and physical structure (2010) Polymer, 51:3213-24. [5] A. Dorfmann, R.W. Ogden, A pseudo-elastic model for the Mullins effect in filled rubber (2004), International Journal of Solids and Structures 41: 1855-1878.

[6] R. W. Ogden, D. G. Roxburgh, A pseudo-elastic model for the Mullins effect in filled rubber, Proc. R. Soc. Lond. A 455 (1999) 2861-2877.

[7] L. Mullins, Softening of rubber by deformation (1969), Rubber Chem. Technol. 42, 339-362.

[8] L. Mullins, N. Tobin, Theoretical model for the elastic behavior of filler-reinforced vulcanized rubbers (1957), Rubber Chem. Technol. 30, 51-571.

[9] G. Chagnon, E. Verron, G. Marckmann, L. Gornet, Development of new constitutive equations for the Mullins effect in rubber using the network alteration theory (2006), International Journal of Solids and Structures, Volume 43, Issues 22-23, Pages 6817-6831.

[10] G. Marckmann, E. Verron, L. Gornet, G. Chagnon, P. Charrier, P. Fort, Comparison of hyperelastic models for rubber-like materials (2002), J. Mech. Phys. Solids 50, 2011-2028.

[11] O. Jaudouin, J. J. Robin, D. Perrin, R. Sonnier, P. Ienny, R. Léger, J. M. Lopez-Cuesta, "Incorporation of Organomodified Layered Silicates and Silica in Thermoplastic Elastomers in Order to Improve Tear Strength", Materials Science Forum, Vol. 714 (2012), pp. 217-227. 
[12] C. G’Sell, A. Coupard. Génie mécanique des caoutchoucs et les élastomères thermoplastiques, chapter "Formulation des élastomères - M.-N. Bouchereau". Apollor, INPL, LRCCP, FIRTECH, 1997. [13] O. Aso, J.I. Eguiazábal, J. Nazábal, The influence of surface modification on the structure and properties of a nanosilica filled thermoplastic elastomer (2007), Composites Science and Technology, Volume 67, Issue 13, Pages 2854-2863.

[14] Bradley Finnigan, Darren Martin, Peter Halley, Rowan Truss, Kayleen Campbell, Morphology and properties of thermoplastic polyurethane nanocomposites incorporating hydrophilic layered silicates, Polymer 45 (2004) 2249-2260.

[15] P.A. Hassan, Gunjan Verma, R. Ganguly, 1 - Soft Materials - Properties and Applications, Functional Materials (2012), pp. 1-59.

[16] O. Jaudouin, Physico-chimie de matériaux à base d'élastomères modifiés hyperélastiques, $\mathrm{PhD}$ Thesis (2011), University of Montpellier 2, 270 p. - Source: http://www.theses.fr/2012MON20036.

[17] K. Haraguchi, M. Ebato and T. Takehisa, "Polymer-Clay nanocomposites Exhibiting Abnormal Necking Phenomena Accompanied by Extremely Large Reversible Elongations and Excellent Transparency", Advanced Materials (2006), 18, pp 2250-2254.

[18] Y.W. Chen-Yang, Y.K. Lee, Y.T. Chen, J.C. Wu, High improvement in the properties of exfoliated PU/clay nanocomposites by the alternative swelling process, Polymer (2007), 48, pp 29692979.

[19] Polyurethane composition having improved tear strength and process for preparation thereof Patent, US Patent (1977), 4,062,825.

[20] K. Vuillaume, Interactions élastomères - charges. Mécanismes de déplacement des molécules adsorbées et co-adsorbées. PhD Thesis (2001), University de Mulhouse. Source: http://www.theses.fr/2001MULH0641.

[21] M.P. Wagner, Reinforcing Silicas and Silicates. Rubber Chemistry and Technology (1976), 49(3), 703-774. doi:10.5254/1.3534979.

[22] Z. Zhenjung, Z. Lina, L. Yang, Effect of the Addition of Toluene on the Structure and Properties of Styrene-Isoprene-Butadiene Rubber/Montmorillonite Nanocomposites (2005), Macromolecular Materials and Engineering, Volume 290, Issue 5, pages 430-437.

[23] Y.W. Chen-Yang, Y.K. Lee, Y.T. Chen, J.C. Wu, High improvement in the properties of exfoliated PU/clay nanocomposites by the alternative swelling process, Polymer 48 (2007) pp. 29692979.

[24] C. Deng, X. Lu, S.B. Zhou, J.X. Wan, S.X. Qu, B. Feng, X.H. Li, Q.Y. Cheng, Mechanism of ultrahigh elongation rate of poly(D,L-lactide)-matrix composite biomaterial containing nano-apatite fillers (2008), Materials Letters, Volume 62, Issues 4-5, Pages 607-610.

[25] Z. Ma, X. Huang, Pi. Jiang, A comparative study of effects of SEBS and EPDM on the water tree resistance of cross-linked polyethylene (2010), Polymer Degradation and Stability, Volume 95, Issue 9, September 2010, Pages 1943-1949. 
[26] X. Wang, S.-I. Pang, J.-he Yang, F. Yang, Structure and properties of SEBS/PP/OMMT nanocomposites, Transactions of Nonferrous Metals Society of China, 16:2 (2006) pp. 524-528.

[27] M.-Q. Zhan, K.-K. Yang, Y.-Z. Wang, Shape-memory poly(p-dioxanone)-poly( $(\varepsilon-$ caprolactone)/sepiolite nanocomposites with enhanced recovery stress, Chinese Chemical Letters 26 (2015), pp. 1221-1224.

[28] Flexible sheets for waterproofing - Determination of resistance to tearing - Part 2: plastic and rubber sheets for roof waterproofing. NF EN 12310-2 February 2001.

[29] W. Kuang, G.A. Facey, C. Detellier, B. Casal, J.M. Serratosa, E. Ruiz-Hitzky, Nanostructured Hybrid Materials Formed by Sequestration of Pyridine Molecules in the Tunnels of Sepiolite. Chem Mater 2003;15:4956-67.

[30] G. Tartaglione, D. Tabuani, G. Camino, Thermal and morphological characterization of organically modified sepiolite. Microporous Mesoporous Mater 2008;107:161-8.

[31] C. del Hoyo, C. Dorado, M. S. Rodríguez-Cruz and M. J. Sánchez-Martín, Physico-chemical study of selected surfactant-clay mineral systems, Journal of Thermal Analysis and Calorimetry (2008), vol. 94, Issue 1, pp. 227-234.

[32] R. Benlikaya, M. Alkan, İ Kaya, Preparation and characterization of sepiolite-poly (ethyl methacrylate) and poly (2-hydroxyethyl methacrylate) nanocomposites, Polym Composite (2009), 30, pp. 1585-1594.

[33] Nabarun Roya and Anil K. Bhowmick, Novel in situ polydimethylsiloxane-sepiolite nanocomposites: Structure-property relationship, Polymer (2010), vol. 51, Issue 22, pp. 5172-5185.

[34] M. Maiti, A.K. Bhowmick, Structure and properties of some novel fluoroelastomer/clay nanocomposites with special reference to their interaction, J. Polym Sci Part B Polym Phys (2006), 44 (1), pp. 162-176. 